\title{
A LANDMARK MATCHING ALGORITHM FOR THE GEOSTATIONARY SATELLITE IMAGES BASED ON MULTI-LEVEL GRIDS
}

\author{
S.Y. Hou ${ }^{1}$, Z.Y. Qin ${ }^{1}$, L. Niu ${ }^{1 *}$, W.G. Zhang ${ }^{1}$, W.T. Ai ${ }^{1}$ \\ ${ }^{1}$ School of Surveying and Urban Spatial Information, Henan University of Urban Construction, \\ Pingdingshan, Henan, China - housaoyang@163.com
}

\begin{abstract}
KEY WORDS: Geostationary Satellite Images, Landmark Matching, Coastline Template, Multi-level Grids, Contour Feature, Feature Matching
\end{abstract}

\begin{abstract}
:
The resolution of geostationary satellite image is not high and the image is covered with clouds. At present, when the extracted feature points are unstable, there are some problems, such as low matching accuracy or even matching failure. In this paper, a landmark matching algorithm is proposed to directly establish the multi-level grids for the image coastline and the coastline template. Through the similarity measure of the multi-level grids, the landmark matching is realized layer by layer. First of all, we've finished cloud detection, establishment of landmark data set, and extraction of image coastline. Then we design and implement the landmark matching algorithm based on multi-level grids. Finally, through analysis from different levels of landmarks and different proportion of cloud cover, the advantages and applicable conditions of this algorithm are given. The experimental results show that: 1) with the increase of cloud cover, the correct rate of landmark matching decreases, but the decrease is small. It shows that the matching algorithm in this paper is stable. Correct matching rate could always be stable at about 75 percent in the fourth level. 2) when the proportion of cloud cover is less than 20 percent, the higher the matching level, the higher the matching accuracy. When the cloud cover is more than 20 percent, the matching accuracy in the fourth level is the highest. This algorithm provides a stable method for the landmark matching of geostationary satellite image.
\end{abstract}

\section{INTRODUCTION}

When the geostationary satellite is in orbit, due to the influence of internal system and external environmental factors, the scanning mirror direction of satellite sensor will change slightly, which will cause geometric distortion of satellite image, and then affect the positioning accuracy. Because of the characteristics of geostationary satellite, such as high orbit, low resolution and cloud influence, the method of landmark matching is often used to correct the direction of geostationary satellite. Landmark matching will encounter the selection of landmarks depending on the distribution of the cloud in the whole disk region. The area without cloud or less cloud is selected as the matching area. Finally, in the landmark area the coastline template and the coastline extracted from geostationary satellite image are matched (Ding, Tong, Qin, 2018).

The essence to the current landmark matching method is to extract accurate feature points, and transform the feature line matching into the feature point matching. Yang Lei studied the method of automatic landmark matching of the FY-2 meteorological satellite image (Yang, Yang, 2009). After selecting the ground feature points, the landmark template and the image were matched by the maximum correlation coefficient method (Yang, Feng, Guo, 2011). Ding Lu proposed a landmark matching method for determining the pointing of three-axis stable geostationary orbit remote sensing satellite. Firstly, the rough matching between landmark data set and image coastline features is realized, and the feature points to be matched are screened. The correlation between gradient direction is taken as the main measure, and the difference of Zernike moment and error ellipse is used as auxiliary parameters to realize fine matching, taking into account the matching success rate and computational efficiency (Ding,
Tong, Qin, 2018). In addition to its application in landmark matching, feature line matching is often used to match multi-source remote sensing images with obvious edges and contours (Yu, Lu, Hu, 2013; Wang, Wang, Li, 2011; Li, Zhang, Zhang, 2019), such as SAR images and optical images(Xiang, Wang, You, 2018;Rui, Wang, Zhang, 2019), infrared images and optical images(Li, Jiang, Xu, 2015; Hu, Wang, Liu, 2013) . In these processes, feature line matching is transformed into feature point matching (Liu, Huo, Han, 2017; Song, 2014) and intensity-based registration (Liang, Liu, Huang, 2014; Moorthil, Sivakumar, 2018).

At present, the key of landmark matching method is to extract enough, uniform and accurate feature points from the satellite image. However, the resolution of the image is not high, the texture is complex, and it is covered with clouds, which leads to the difficulty of accurate extraction of feature points, the large amount of computation, the small number of high quality feature points, the fewer feature point pairs that can be used for landmark matching. Eventually, it leads to the low matching accuracy and even the failure of matching when the feature extraction is unstable. Through the analysis, it is found that the overall spatial structure of the feature line is much more stable than the feature point under the condition that the feature line is partially occlusive and the resolution is not high. Therefore, it is possible to achieve stable landmark matching by studying the whole spatial position similarity of the feature line.

In this paper, the multi-level grids are established directly for the whole contour feature of the coastline without extracting the feature points. Through the similarity measure of the multi-level grids, the landmark matching is realized layer by layer, trying to find a robust landmark matching method, which provides a stable method for the landmark matching of the geostationary satellite images.

* Corresponding author 


\section{DATA PREPARATION}

\subsection{Cloud Detection}

The geostationary orbit satellite data used in this paper comes from the National Satellite Meteorological Center. It is FY-4A image, level 1 ARGI data, HDF format, $2000 \mathrm{~m}$ resolution, and the product name is full disk $2 \mathrm{KML} 1$ data. After downloading the data, the HDF data is converted to raw format by a piece of software called FengYunToolkit. This paper only uses NOMChannel01 and NOMChannel03, and then we use C++ language programming to read the raw format data and convert it into bmp format data with a size of $5496 \times 5496$ image. The image is unbiased, so it is translated 10 pixels to the right and 5 pixels down. The NOMChannel03 after translation is the data to be matched. According to the histogram of NOMChannel01 as shown in figure 1.a, we set a threshold to detect cloud as shown in figure 1.b. , and the threshold is greater than 55. We get fusion image of NOMChannel01 and NOMChannel03 as shown in figure 1.c, where the values of the cloud pixels are 255 and the other pixels are the same as those in the NOMChannel03.

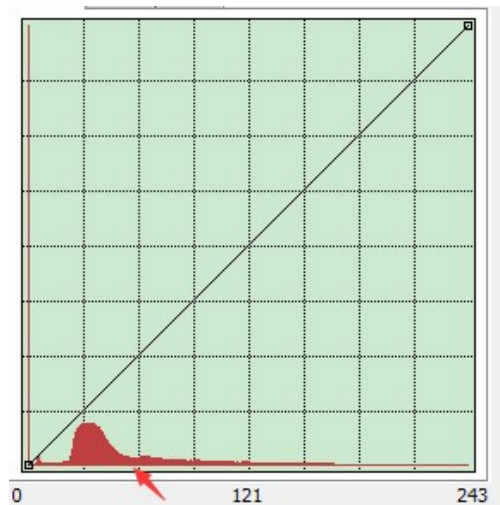

Figure 1.a. Histogram

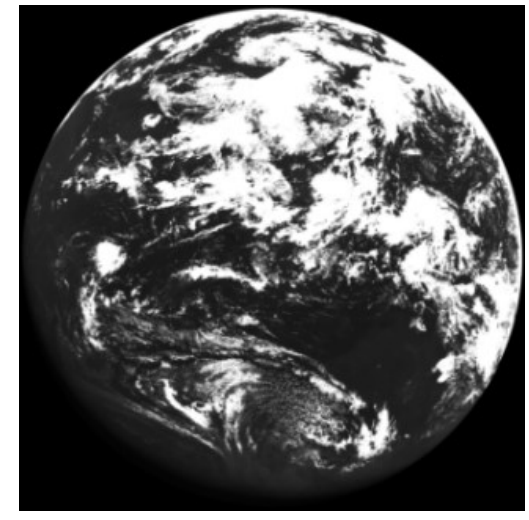

Figure 1.b. Detection cloud

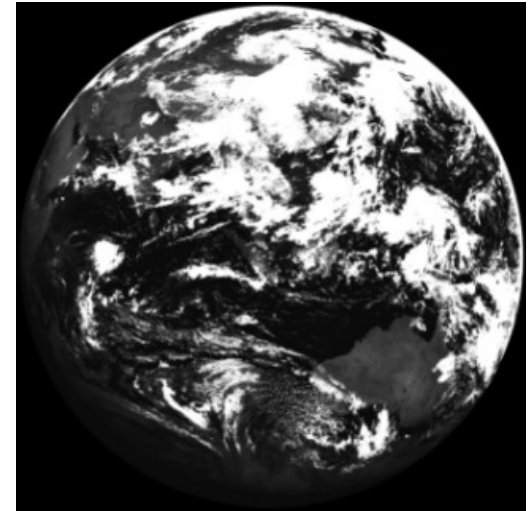

Figure 1.c. Fusion image

\subsection{Establishment of Landmark Data set}

First of all, according to the histogram of the fusion image, the fusion image (Figure 1.c.) is classified as cloud (white), water (blue) and land (green), as shown in Figure 2. (left). Then, combining the coastline template (red), the restricted quad-tree grids are established according to the classified image, and the initial maximum grid level is six. In the experimental part of this paper, the grid level is discussed. The size of pixels in a landmark is $87 \times 87$. Because the resulting image needs to be stored in bmp format, the number of pixels in the row and column of the image is required to be a multiple of 4 , so the size of an image is $88 \times 88$. Finally, taking a grid as a unit, the ratio of white, blue, green and red pixels is counted, and the initial threshold values are set to $<0.2,>0.01,>0.1,>0.001$. A grid satisfying the above four conditions is a landmark, as shown in figure 2., in which the yellow grid is the landmark area. The right figure shows the landmark superimposed on the coastline template. It is a raster data, with a resolution of 2000 meters and the size of $5496 \times 5496$. The coastline template is the reference data

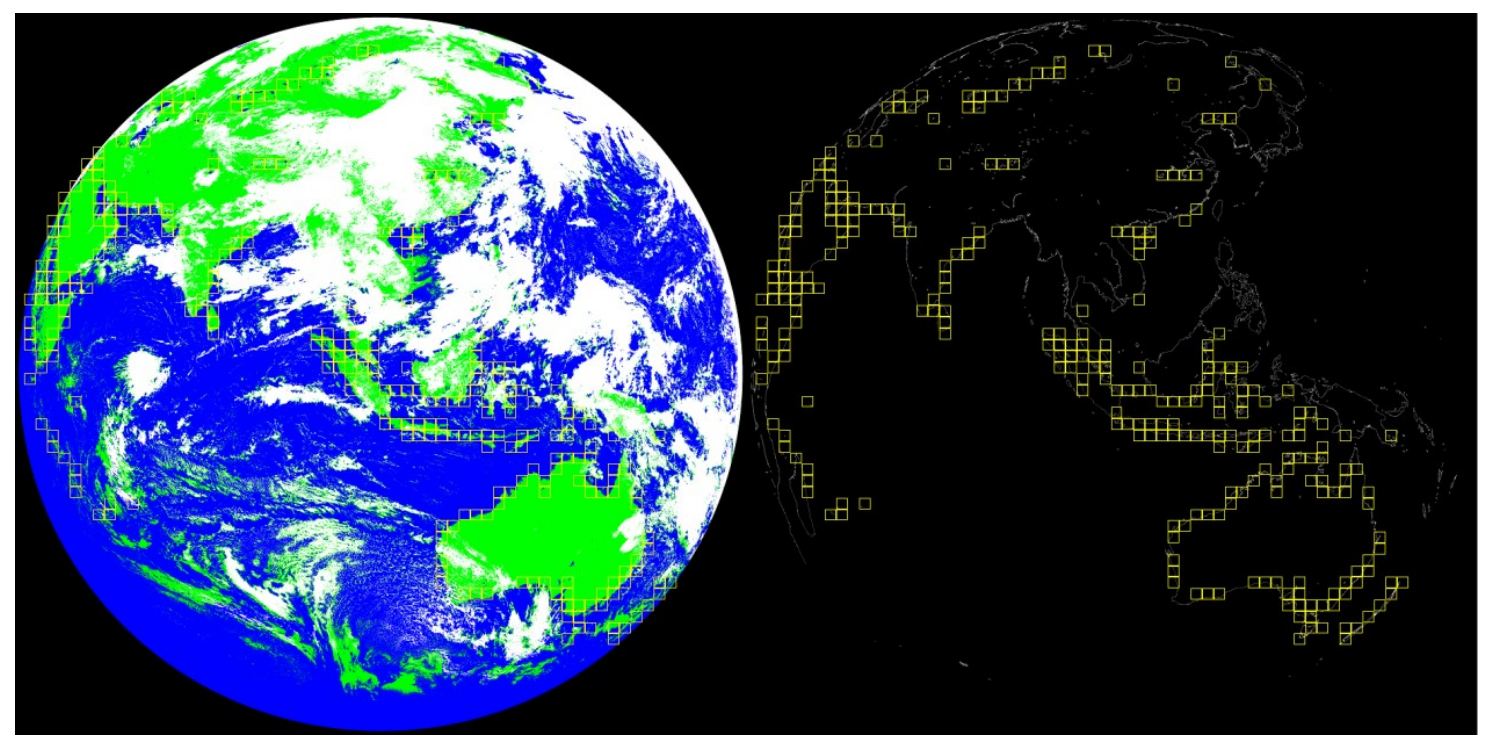

Figure 2. Landmark data set (yellow grid is landmark, left image is classified image superimposed landmark, right image is the coastline template superimposed landmark) 


\subsection{Image coastline detection}

Coastline detection includes three steps: corrosion of sea-land boundary area, removal of small patches and extraction of image coastline. Firstly, each blue pixel is traversed and corroded by a $3 \times 3$ grid. If there is a green pixel in the $3 \times 3$ grid, the pixel is retained, otherwise it is assigned to 0 . Then, the small patch is removed and the window of $3 \times 3$ is used to traverse all the pixels. If the central pixel is different from the surrounding pixel, its pixel value is changed to the value that appears the most frequently in the $3 \times 3$ window. Finally, assign the blue pixel to 255 (white) and the other pixel to 0 (black), as shown in figure 3.a. The coastline template clipped by landmarks is shown in figure 3.b. Through the comparison, it can be seen that due to the cloudy occlusion, some of the coastline extracted from the image are missing or inconsistent, and some of them are more missing.

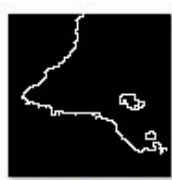

4_42.bmp

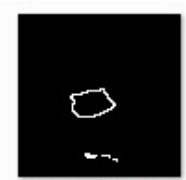

5_27.bmp

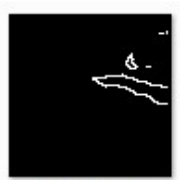

6_23.bmp

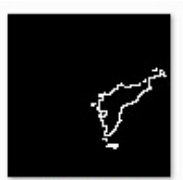

7_19.bmp

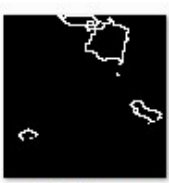

7_22.bmp

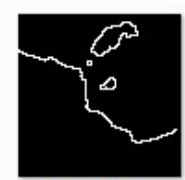

42_51.bmp

Figure 3.a. Image coastline extracted by this method

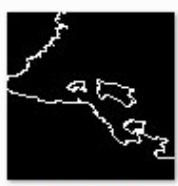

4_42.bmp

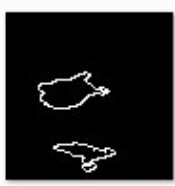

5_27.bmp

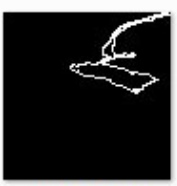

6_23.bmp

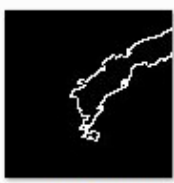

7_19.bmp

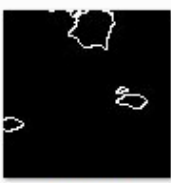

7_22.bmp

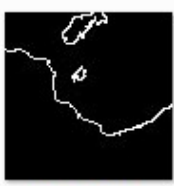

42_51.bmp

Figure 3.b. Coastline template data clipped by the landmarks

\section{LANDMARK MATCHING ALGORITHM AND EXPERIMENT BASED ON MULTI-LEVEL GRIDS}

\subsection{Matching Algorithm}

The data to be matched are the image coastline extracted in this paper and the coastline template. The template data is the reference image, and the image coastline is the image to be corrected. This algorithm is divided into the following six steps,and the experimental results are shown in figure 4 .

In the first step, the image landmark data set is traversed, and the maximum subdivision level of the basic grid is twelve, which is the maximum effective partition level of image with the size of $5496 \times 5496$. The restricted quad-tree algorithm is used to divide the block data recursively. The partition condition is as follows: there are pixels with the value of 255 in the block.

In the second step, the image coastline grids are extracted by restricted quad-tree algorithm. Grids are processed separately from the 7 th to $12^{\text {th }}$ level , and the translation offset of hierarchical data is set up. At the same time, the restricted quad-tree is also established for the coastline template, and the hash table of the coastline template is established. The key is grid code, consists of level, row, and column, and the value is node class.

In the third step, the image coastline grids in the $i^{\text {th }}$ level $(7 \leq i \leq 12)$ are traversed, and nine kinds of translation images are established in the $3 \times 3$ window. Compared with the coastline template grid, the similarity is the highest when the grids coincide the most. The translations in the $\mathrm{x}$ and $\mathrm{y}$ directions are stored in the offxs and offys vector respectively, and inserted at the beginning of the vector until the $12^{\text {th }}$ level.
When dealing with the $i^{\text {th }}$ level landmark, the cumulative translation needs to be added from 7 to $(i-1)$.

In the fourth step, in the $\mathrm{x}$ and $\mathrm{y}$ direction translation pixel values of the image are calculated. The specific expressions are as follows. The off $X$ is the translation in the $\mathrm{X}$ direction, and off $Y$ is the translation in the $\mathrm{Y}$ direction. The variable $r$ is the number of rows in the image divided by the number of rows in the grid, indicating the number of pixels occupied by a single grid. In this paper, the grid is divided into the $12^{\text {th }}$ levels, so the number of rows is 4096 . The size of the image is $5496 \times 5496$, so $r$ is $5496 / 4096$.

$$
\begin{aligned}
& \text { off } X=\sum_{i=0}^{5} \text { offxs }[i] \times \operatorname{pow}(2.0 f, i) \times r \\
& \text { off } Y=\sum_{i=0}^{5} \text { offys }[i] \times \operatorname{pow}(2.0 f, i) \times r
\end{aligned}
$$

where $\quad$ off $X=$ translation in the $X$ direction off $Y=$ translation in the $Y$ direction off $x$ s $=$ translation vector in $\mathrm{X}$ direction offys $=$ translation vector in $\mathrm{Y}$ direction $i=$ landmark level $r=$ image size divided by grid size

In the fifth step, off $X$ and off $Y$ are used for image translation, and then the translated image landmark data is merged with the coastline template. The fusion image is shown in figure 5.b,and the image before fusion is shown in figure 5.a.

In step 6, cycle through the above five steps until all landmark matches are completed. 

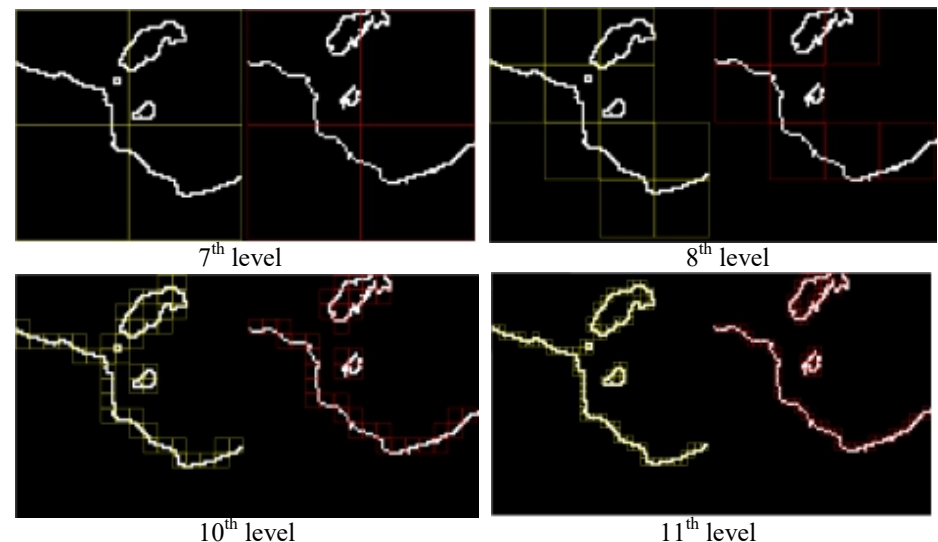

$11^{\text {th }}$ level
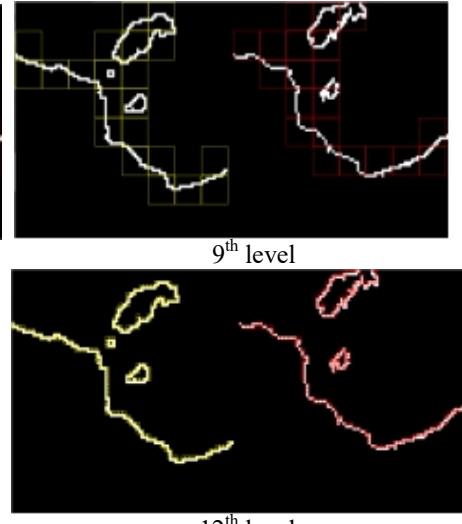

$12^{\text {th }}$ level

Figure 4. Multi-level grids matching algorithm (take the image in column 51 of line 42 as an example)

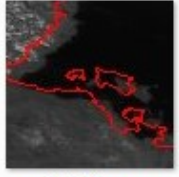

4_42.bmp

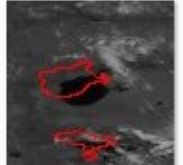

5_27.bmp

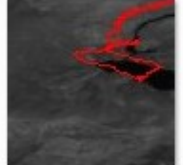

6_23.bmp

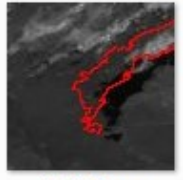

7_19.bmp

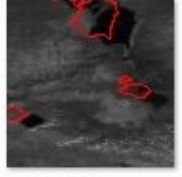

7_22.bmp

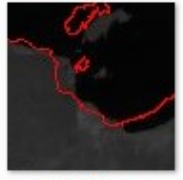

42_51.bmp

Figure 5.a. Fusion images before matching

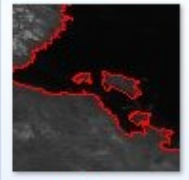

4_42.bmp

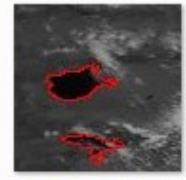

5_27.bmp

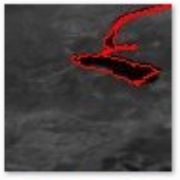

6_23.bmp

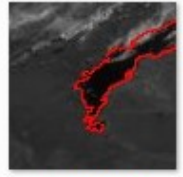

7_19.bmp

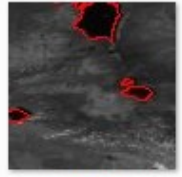

7_22.bmp

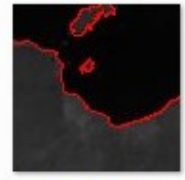

42_51.bmp

Figure 5.b. Fusion images after matching

\subsection{Matching Experiment}

Taking the image in column 51 of line 42 as an example, the multi-level grids from the $7^{\text {th }}$ level to the 12th level is shown in Figure 4., where the left picture is the image coastline, and the right picture is the coastline template. Through the calculation of the algorithm in this paper in section 3.1, the translation vector in the $\mathrm{X}$ direction is $(0,0,0,-1,0,0)$, and in the $\mathrm{Y}$ direction it is $(0,0,1,-1,0,0)$. The translation vector is substituted into the formula of the fourth step in section 3.1. The translation in the $\mathrm{X}$ direction is negative ten, and the translation in the $\mathrm{Y}$ direction is negative five. In other words, the image in column 51 of line 42 needs to translate ten pixels to the left and move up five pixels. The image after translation is shown in figure 5.b, in which the last image is the image in column 51 of line 42 , and the fusion image before matching is shown in figure 5 .a.

\section{EXPERIMENTAL ANALYSIS}

The accuracy extraction of image coastline, cloud cover of the landmark and landmark level are the most important factors that affect the matching effect of the algorithm in this paper. At present, the coastline extracted can basically meet the requirements of the algorithm, and it can be optimized in the future research. In this paper we mainly discuss the influence of two parameters, namely, the cloud cover and the level of the landmark. The experimental results show that the matching effect is stable when the landmark level is from three to five in Table 1.. The cloud cover of the landmark discussed in this paper is $0.05,0.1$, $0.2,0.3,0.4,0.5,0.6,0.7$, and 0.8 . The specific experimental results are shown in Table 1. In this table, N3, N4, N5 represent the number of the selected landmarks, and the size of landmark in the third to fifth level is $692 \times 692,348 \times 348$, and $176 \times 176$. T3, T4, T5 represent the matching time of all selected landmarks in the third to fifth level. The correct matching rate of landmarks refers to the proportion of landmarks whose translation error does not exceed one pixel. The standard translations are 10 pixels and 5 pixels in the $\mathrm{X}$ direction and the $\mathrm{Y}$ direction.

\subsection{Analysis of level change of landmarks}

From the analysis of matching accuracy, the lower the landmark level is, the larger the landmark size is, and the matching accuracy tends to increase. But when the cloud cover exceeds 20 percent, the matching accuracy in the fourth level is better than that in the third level. It indicates that the third level is greatly affected by the cloud, and the coastline of the third level is more broken after being obscured by the cloud. The number of grids in the third level subdivided to the higher level is more than that in the fourth level when the restricted quad-tree is established. It can be confirmed by the time ratio of single landmark matching. When the cloud cover is less than 20 percent, the higher the landmark level is, the higher 
the matching accuracy is. The higher the landmark level is, the higher the matching accuracy is. When the cloud cover is more than 20 percent, the matching accuracy of the $4^{\text {th }}$ level is the highest.

From the analysis of matching time efficiency, when cloud cover is less than 10 percent, the higher the level of landmark is, the less the matching time is. When the cloud cover is more than 10 percent, the matching time of the 4 th level is the least.

From the analysis of landmark selection flexibility, the higher the level of landmark is, the smaller the size of landmark is. And the more the number of selected landmarks is, the more scattered the distribution of landmarks is. When the selected landmarks are used in a certain area to match, the more optional landmarks are, the greater the possibility of finding the most suitable matching landmarks in all positions of the whole disk is.

To sum up, when selecting the level of the landmark, the size should be appropriate. The offset of the whole earth disk of the actual geostationary orbit satellite image is not always the same. When the local landmark area is too large, the offset of different positions of the single landmark may vary greatly, and it is not appropriate to correct any offset. If the landmark area is too small, although the number of selected landmarks is large, it is vulnerable to cloudy influence, and the matching accuracy will be greatly reduced. From the experimental results of this paper, when the landmark is selected in the fourth level, the efficiency and stability of the algorithm are the best.

\begin{tabular}{|c|c|c|c|c|c|c|c|c|c|c|c|c|c|c|c|c|}
\hline \multirow[t]{2}{*}{$\begin{array}{l}\text { Cloud } \\
\text { ratio }\end{array}$} & \multicolumn{3}{|c|}{$\begin{array}{l}\text { Number of } \\
\text { selected } \\
\text { landmarks }\end{array}$} & \multicolumn{3}{|c|}{$\begin{array}{l}\text { Matching total time } \\
\text { (Unit:ms) }\end{array}$} & \multicolumn{3}{|c|}{$\begin{array}{c}\text { Correct } \\
\text { matching rate } \\
\text { (Unit:\%) }\end{array}$} & \multicolumn{3}{|c|}{$\begin{array}{l}\text { Single landmark matching } \\
\text { time (Unit:ms) }\end{array}$} & \multicolumn{2}{|c|}{$\begin{array}{l}\text { Time ratio for } \\
\text { single landmark } \\
\text { matching }\end{array}$} & \multicolumn{2}{|c|}{$\begin{array}{l}\text { Time ratio for } \\
\text { the full disk } \\
\text { matching }\end{array}$} \\
\hline & N3 & N4 & N5 & $\mathrm{T} 3$ & $\mathrm{~T} 4$ & T5 & r3 & $\mathrm{r} 4$ & r5 & $\mathrm{t} 3$ & $\mathrm{t} 4$ & t5 & $\mathrm{t} 3 / \mathrm{t} 4$ & $\mathrm{t} 4 / \mathrm{t} 5$ & $\mathrm{~T} 3 / \mathrm{T} 4$ & T4/T5 \\
\hline$<=0.05$ & 4 & 15 & 44 & 713288 & 749803 & 655710 & 100 & 73 & 57 & 178322 & 49987 & 14903 & 3.57 & 3.35 & 0.95 & 1.14 \\
\hline$<=0.1$ & 5 & 23 & 61 & 774035 & 903186 & 744919 & 100 & 74 & 56 & 154807 & 39269 & 12212 & 3.94 & 3.22 & 0.86 & 1.21 \\
\hline$<=0.2$ & 10 & 30 & 90 & 1490168 & 1110649 & 970140 & 90 & 73 & 52 & 149017 & 37022 & 10779 & 4.03 & 3.43 & 1.34 & 1.14 \\
\hline$<=0.3$ & 13 & 39 & 108 & 2363780 & 1247195 & 1061556 & 69 & 77 & 51 & 181829 & 319 & 9829 & 5. & 3.25 & 1.90 & 1.17 \\
\hline$<=0.4$ & 15 & 45 & 132 & 2493144 & 1437006 & 1189308 & 73 & 78 & 45 & 166210 & 31933 & 9010 & 5.20 & 3.54 & 1.73 & 1.21 \\
\hline$<=0.5$ & 18 & 48 & 138 & 3127595 & 1490644 & 1232806 & 67 & 75 & 49 & 173755 & 31055 & 8933 & 5.60 & 3.48 & 2.10 & 1.21 \\
\hline$<=0.6$ & 19 & 56 & 147 & 3762046 & 1675885 & 1260377 & 63 & 70 & 46 & 198002 & 29927 & 8574 & 6.62 & 3.49 & 2.24 & 1.33 \\
\hline$<=0.7$ & 19 & 61 & 153 & 3762046 & 1722910 & 1284366 & 63 & 66 & 46 & 198002 & 28244 & 8395 & 7.01 & 3.36 & 2.18 & 1.34 \\
\hline$<=0.8$ & 22 & 62 & 157 & 3349888 & 1727696 & 1293732 & 64 & 66 & 45 & 152268 & 27866 & 8240 & 5.46 & 3.38 & 1.94 & 1.34 \\
\hline
\end{tabular}

Table 1. Parameter of landmarks matching under different cloud cover

\subsection{Analysis of cloud change in a landmark}

From the analysis of matching accuracy, with the increase of cloud cover, the correct rate of landmark matching decreases, but the decrease is not large, which shows that the matching algorithm in this paper is stable. When the cloud cover exceeds 50 percent, there is still about 70 percent correct matching rate. Especially in the fourth level, the correct matching rate of landmarks can always be stable at about 75 percent.

With the increase of cloud cover, the matching time of one landmark decreases gradually. While with the increase of cloud cover, the matching time fluctuates, sometimes even increases. The clouds block the image and complicate the landmark. It not only affects the spatial distribution characteristics of image coastline, but also may be optimized or complicated. If the effect of cloud is to simplify, it will reduce the matching time, such as the forth level and the fifth level. If the coastline is broken, it will not only increase the matching time, but also reduce the matching accuracy, such as the third level.

To sum up, the forth level is the least affected by cloud, the highest matching accuracy and the most stable landmark matching level. When the optimal the landmark could not be found in some areas in the forth level, the fifth level landmark matching can be considered.

\section{CONCLUSIONS}

In this paper, multi-level grids are established directly for the image coastline, and the landmark matching is realized by the similarity measure of the multi-level grid. The multi-level grid has the dual functions of feature representation and feature description, which provides a new idea for the matching of geostationary orbit satellite images. The experimental results are as follows:

1) For the geostationary orbit satellite images with cloud cover and complex texture, the method of global matching of the coastline can achieve stable landmark matching without extracting feature points. The matching method without control points can avoid complex operations such as feature point pair extraction and optimization, and reduce the complexity of computation and algorithm.

2) When the cloud cover is less than 20 percent, the higher the landmark level is, the higher the matching accuracy is. When the cloud cover is more than 20 percent, the highest matching accuracy is in the forth level. When the cloud cover is less than 10 percent, the higher the landmark level is, the less matching time is when the same size image is matched. When the cloud cover is more than 10percent, the matching time of the fourth level is the least. 
3) With the increase of cloud cover, the correct rate of landmark matching decreases, but the decrease is small, which shows that the matching algorithm in this paper is stable. When the cloud cover exceeds 50 percent, there is still about 70 percent correct matching rate. Especially in the fourth level, the correct matching rate of landmarks can always be stable at about 75 percent.

4) The forth level is the least affected by cloud, the highest matching accuracy and the most stable landmark matching level. When the optimal matching landmark could not be found in some areas, the fifth layer landmark matching can be considered.

Next, the geometric error distribution of the whole disk will be studied to ensure the optimal landmark matching of the whole disk. The extraction and optimization of the contour feature will continue to be studied, and the algorithm of large error will be improved to further improve the matching accuracy of the algorithm in this paper. The influence of clouds on the spatial distribution characteristics of the image coastline will be studied under different sizes of the landmarks, which will be further optimized.

\section{ACKNOWLEDGEMENTS}

This work was supported by the National Natural Science Foundation of China under Grants 41701454, by Scientific Research Capability Upgrading Project of Henan University of Urban Construction under Grants 2017YY022, and by Youth Backbone Teacher Funding Project of University of Urban Construction of Henan Province under Grant YCJQNGGJS201806.

\section{REFERENCES}

Ding, L., Tong, X.C, Qin, Z.Y., 2018. Landmark matching method about pointing to the ground of three-axis stabilization geostationary remote sensing satellite. Journal of surveying and Mapping, 47 (11), $1506-1517$.

Hu, Y.L., Wang, L., Liu R., 2013. A coarse-to-fine registration method for satellite infrared image and visible image. Spectroscopy and Spectral Analysis, 33 (11), $2968-2972$.

Li, K., Zhang, Y.S., Zhang, Z.C., 2019. A coarse-to-fine registration strategy for multi-sensor images with large resolution differences. Remote Sensing, 11 (7), 470- 497.
Li, Z.H., Jiang G.H., Xu S.N., 2015. Image registration algorithm for infrared and visible images based on contour polygon fitting. Systems Engineering and Electronics, 37 (12), 2872 - 2878.

Liang, J.Y., Liu, X.P., Huang, K.N., 2014. Automatic registration of multi-sensor images using an integrated spatial and mutual information (SMI) metric. 2014 IEEE Trans. Geosci. Remote Sens, 52(1), 603-615.

Liu, K.J., Huo, H.T., Han, H., 2017. Image registration using geometric constrain between contour points. Remote sensing information, 32 (03), 128-132.

Moorthil, S.M., Sivakumar, R., 2018. Comparison of contour feature based and intensity based insat-3D met images coregistration for subpixel Accuracies. Ictact Journal on Image and Video Processing, 08(04):1731-1738.

Rui, J., Wang, C., Zhang H., 2019. Multi-sensor SAR image registration based on object shape. Remote Sensing, 8 (11), 923-933.

Song, Z.L., 2014. Automatic approach based on point and curve features for multimodal remote sensing image registration. Remote Sensing of Land and Resources, 26 (03), 48-54.

Wang, H.Y., Wang, C., Li, P., 2011. Review of multi-source remote sensing image registration techniques. Computer Engineering, 37 (19), 21-25.

Xiang, Y. M., Wang, F., You, H.J., 2018. An automatic and novel SAR image registration algorithm: A case study of the Chinese GF-3 satellite. Sensor, 18 (2), 672-694.

Yang, L. Yang Z.D., 2009. The automated landmark navigation of the polar meteorological satellite. Journal of Applied Meteorology, 20 (3), 329-336.

Yang, L., Feng, X.H., Guo, Q., 2011. Automatic geometric precision correction of Fengyun-2 meteorological satellite imagery. Computer Engineering and Applications, 47 (03), 202-206.

Yu, X.C., Lu, Z.H., Hu, D., 2013. Review of remote sensing image registration techniques. Optical and Precision Engineering, 21 (11), 2960-2972. 